\title{
Service Science - Potenziale für die Weiter- entwicklung der Dienstleistungsgesellschaft
}

Die Industrielle Revolution hat im 19. Jahrhundert die wirtschaftlichen Machtverhältnisse und die Arbeitsgestaltung dramatisch verändert - profitieren konnten diejenigen Individuen, Unternehmen und Volkswirtschaften, die frühzeitig innovative wissenschaftliche Erkenntnisse erworben, verbreitet und umgesetzt haben. Stehen wir aber nicht gerade jetzt am Beginn einer neuerlichen, mindestens ebenso mächtigen „Service-Revolution“? Werden nicht wiederum innovative Kenntnisse und Fähigkeiten den (globalen) Wettbewerb entscheiden? Müssen wir nicht neue dienstleistungspolitische Wege einschlagen, um den damit verbundenen Herausforderungen begegnen zu können und brauchen wir hierzu nicht eine Dienstleistungswissenschaft?

\section{Dienstleistungswissen- schaft - Phantom oder Phänomen?}

Obwohl Dienstleistungen einen seit Jahrzehnten zunehmenden Anteil volkswirtschaftlicher Wertschöpfung und Beschäftigung ausmachen, konnte man lange Zeit nicht von einer eigenständigen, konsistenten und ihrer selbst bewussten Dienstleistungsforschung oder gar Dienstleistungswissenschaft sprechen. Zwar wird seit Langem zu Dienstleistungen geforscht - etwa bereits in der Frühzeit der Soziologie zur Arbeit im Bereich personenbezogener Dienstleistungen (z. B. MacLean 1899) oder in den Wirtschaftswissenschaften in bestimmten Branchen wie etwa Finanzdienstleistungen, Tourismus oder Gesundheitsdienstleistungen (Stauss 2008, S. 58). Jedoch erst in jüngerer Zeit beginnt man, Forschung zu Dienstleistungen als Dienstleistungsforschung zu begreifen und diese gezielt zu gestalten.

Einen wichtigen Schritt in diese Richtung stellte die in den 1990er Jahren einsetzende Förderung nationaler Dienstleistungsforschung durch das Bundesministerium für Bildung und Forschung (BMBF) dar. National und international erwuchsen Bestrebungen, eine Service Science zu etablieren (Spohrer et al. 2007) - in Deutschland wurden in der Initiative „Partner für Innovation" die Potenziale einer eigenen Dienstleistungswissenschaft diskutiert (Stauss et al. 2008). An den Universitäten, aber auch an Fachhochschulen und dualen Bildungseinrichtungen, hat sich nicht nur die Zahl spezifischer Lehrstühle für
Dienstleistungsmanagement vervielfacht, sondern es sind auch in Deutschland Forschungsinstitute entstanden, die über die Betriebswirtschaft hinaus interdisziplinäre Dienstleistungsforschung vorantreiben. ${ }^{1}$ Neue Ausbildungs- und Studiengänge auf Bachelor- und Masterniveau entstehen vielerorts; eine Vielzahl wissenschaftlicher Kongresse schießt aus dem Boden; eigene Fachzeitschriften werden gegründet und gewinnen Reputation und Leserschaft. ${ }^{2}$

Dennoch fällt es schwer, Zielrichtungen und Gemeinsamkeiten dieser Initiativen zu erkennen und zu artikulieren. Ein gerüttelt Maß Unklarheit wird bereits durch die unterschiedlichen Begriffsverständnisse hervorgerufen, mit denen eine potenzielle Dienstleistungswissenschaft als Ergänzung für die jeweils eigene Disziplin reklamiert wird:

- In der Betriebs- und Volkswirtschaftslehre werden als ihr Gegenstand Dienstleistungen verstanden, die in unscharfer Abgrenzung als Gegenstück zu Produkten gesehen werden. Demzufolge werden Ansätze der Entwicklung, Produktion und Vermarktung untersucht, die den spezifischen Eigenschaften von Dienstleistungen Rechnung tragen, z. B. der Immaterialität oder der Integration des Kunden (Engelhardt et al. 1993; Kleinaltenkamp 2001).

- In den Sozialwissenschaften finden sich dienstleistungsrelevante Forschungen in einer Vielzahl von Disziplinen und Teildisziplinen. Beispielsweise bemüht sich die Arbeits- und Industriesoziologie um Fragen des Strukturwandels der Arbeit im Tertiarisierungsprozess (z. B. Jacobsen 2010), um das Besondere der Arbeitsbedingungen und des Arbeitshandelns im Dienstleistungsbereich (z.B. Voswinkel 2005), um kritische Perspektiven auf
Dienstleistungsarbeit (Korczynski/MacDonald 2009) und vieles andere mehr.

- Die Informatik wiederum bezeichnet als Service eine programmtechnisch realisierte Funktionalität mit bestimmten Eigenschaften (Papazoglou/Georgakopoulos 2003) - einen Dienst, der aber in den angelsächsischen Fachtermini unglücklicherweise nicht einfach von einer Dienstleistung unterscheidbar ist, aber in vielen Fällen eine eben solche darstellt.

Die Befürworter der sogenannten „Service-Dominant Logic“ (Vargo/Lusch 2004, 2008) stellen neuerdings die gemeinsame Wertschöpfung mehrerer beteiligter Partner in den Mittelpunkt und verstehen als Service die Anwendung der eigenen Ressourcen und Fähigkeiten innerhalb ei-

\footnotetext{
1 So z.B. das Fraunhofer-Institut für Arbeitswirtschaft und Organisation (IAO) in Stuttgart, das Karlsruhe Service Research Institut (KSRI) am Karlsruhe Institute of Technology (KIT), das Center for Leading Innovation \& Cooperation (CLIC) in Leipzig oder das e-Finance Lab an der GoetheUniversität in Frankfurt.

2 Z. B. das Journal of Service Research, das Journal of Service Science and Management und viele andere. Für eine detaillierte Darstellung der Entwicklungen siehe Satzger/Ganz et al. (2010, S. 13ff.).
}

Gerhard Satzger, Prof. Dr., Karlsruhe Service
Research Institute, Karlsruhe Institute of
Technology (KIT). Arbeitsschwerpunkte:
Gestaltung und Management IT-unterstützter
Dienstleistungen.
e-mail: gerhard.satzger@kit.edu
Wolfgang Dunkel, Dr., Institut für Sozial-
wissenschaftliche Forschung (ISF) München.
Arbeitsschwerpunkte: Interaktive Arbeit,
Personenbezogene Dienstleistungen,
Qualifikation, Arbeit und Subjekt.
e-mail: wolfgang.dunkel@isf-muenchen.de


ner Wertschöpfungspartnerschaft, eines "Service-Systems“ (Maglio et al. 2006). Im folgenden Abschnitt 2 wird zunächst dieser Perspektivenwechsel näher beleuchtet, der durchaus helfen kann, die obigen Sichtweisen zu integrieren. Der dadurch entstehende Untersuchungsgegenstand des Dienstleistungssystems (Service System) wird in Abschnitt 3 näher beschrieben. In Abschnitt 4 wird auf kritische Einwände zur Service Science Bezug genommen und es werden einige Vorschläge zu ihrer Weiterentwicklung formuliert. Schließlich werden die Potenziale einer Service Science für Wirtschaft und Gesellschaft diskutiert (Abschnitt 5). ${ }^{3}$

\section{Der Perspektivenwechsel: von der Produktionslogik zur gemeinsamen Wert- schöpfung}

Dass wegen der Heterogenität der genannten Begriffsverständnisse eine gewisse Verwirrung entsteht, verwundert nicht. Andererseits böte eine einheitlich verstandene und allgemein akzeptierte Dienstleistungswissenschaft eine zentrale wissenschaftliche Basis für Dienstleistungsforschung, Dienstleistungswirtschaft und Dienstleistungspolitik. Versuche, über verschiedene Disziplinen hinweg zu einem gemeinsamen Verständnis zu gelangen, existieren bereits - sind aber naturgemäß Gegenstand intensiver und durchaus auch kontroverser Diskussionen, ${ }^{4}$ auf die in Abschnitt 4 näher eingegangen wird. Angestoßen durch die IBM-Initiative „Service Science, Management, and Engineering (SSME)“ (Spohrer et al. 2007) brachte das Cambridge White Paper einen ersten konzertierten Vorschlag zutage (IfM/ IBM 2008); eine deutsche, interdisziplinäre Expertenrunde erarbeitete im Auftrag der Forschungsunion Wirtschaft-Wissenschaft eine Perspektive und Handlungsempfehlungen (Satzger/Ganz et al. 2010). Die neu entstandene deutsche Initiative „3sR - Social Science Service Research“"5 bündelt und vernetzt sozialwissenschaftliche Kompetenzen der Dienstleistungsforschung (Dunkel/Bienzeisler 2011).

Eine Gemeinsamkeit dieser neuen Ansätze ist die Abkehr vom sogenannten „value-in-exchange“ Konzept (Vargo/ Lusch 2004), ${ }^{6}$ nämlich der traditionellen produktionsorientierten Vorstellung, dass geschaffener Wert quasi in ein Produkt implantiert und mit diesem an den Kunden übertragen wird. Denn mehr und mehr wird deutlich, dass die Kooperation mit dem Kunden einen wesentlichen Faktor der Wertschöpfung darstellt und untrennbar mit dieser verbunden ist - die traditionelle Herausforderung für Dienstleistungen und in der Tat für zahlreiche am Markt beobachtbare Leistungen, die zumindest teilweise traditionelle Dienstleistungseigenschaften besitzen (Lösungen, ProduktService-Systeme, Hybride Produkte, etc.). Stattdessen fokussieren diese Ansätze auf den durch die Leistungsbeziehung insgesamt geschaffenen Mehrwert („Valuein-Use") für die beteiligten Partner. Der Beitrag eines Partners besteht dann in den Kenntnissen, Fähigkeiten und Ressourcen, die er für diese „projektartige“ Zusammenarbeit einbringt; die Aktivität der Einbringung ist der Service - ungeachtet dessen, ob ein Leistungsgeber diese zur Fertigung eines Produktes oder zur Erbringung einer traditionellen Dienstleistung in Anspruch nimmt.

Die Perspektive der gemeinsamen Wertschöpfung stellt eine gravierende Änderung des bestehenden Paradigmas dar: Sie bedeutet, dass alle Transaktionen Service-Transaktionen sind (Teboul 2006), allerdings in unterschiedlichem Maße die „gemeinschaftliche Wertschöpfung“ zum Tragen kommt - abhängig vom Grad der Interaktion der Partner und der Individualität des Vorhabens: Während z. B. beim Kauf einer Normschraube die Interaktion mit dem Kunden sich in der Spezifikation des Kundenwunsches erschöpft, sind Unternehmensberatungsleistungen ebenso wie pflegerische und erzieherische Dienstleistungen stark durch Interaktion und individuelle Gestaltung geprägt. Damit stellt das neue Paradigma diejenigen Charakteristika in den Mittelpunkt, die aus wirtschaftswissenschaftlicher Betrachtung den Kern von Dienstleistungen und den Hauptansatzpunkt für eine Separierung von Produkten und Dienstleistungen bilden. Anstatt aber eine artifizielle Trennung von Leistungen exklusive bzw. inklusive dieser Charakteristika herbeizuführen und sich auf Letztere, die Dienstleistungen, zu konzentrieren, rücken nunmehr die Charakteristika selbst in den Blickpunkt. Damit wird insbesondere der Tatsache Rechnung getragen, dass Produkte und Dienstleistungen nicht Ge- gensätze, sondern lediglich Ausprägungen eines Kontinuums sind (Engelhardt et al. 1993). Gleichzeitig wird auch die Bedeutung der Informationsverarbeitung und Telematik deutlich: Nur durch deren breite Anwendung können Interaktion zwischen räumlich entfernten Wertschöpfungspartnern und Individualität über eine große Anzahl von Partnern hinweg gewährleistet werden.

Unter einer sozialwissenschaftlichen Perspektive wird zudem erkennbar, dass Prozesse gemeinsamer Wertschöpfung vor allem auch soziale Prozesse sind. Denn Dienstleistungsbeziehungen sind generell dadurch gekennzeichnet, dass komplexe soziale Prozesse in Gang gesetzt, aufrechterhalten und auch wieder abgeschlossen werden müssen, um den Gegenstand der Dienstleistung realisieren zu können. Diese komplexe, interaktive Arbeit ${ }^{7}$ erbringen Dienstleistungsgeber und Dienstleistungsnehmer gemeinsam: Sie ist deshalb notwendig, weil aus der Sicht einer soziologischen Theorie strategischen Handelns in Dienstleistungsbeziehungen systematisch Probleme auftreten, da Dienstleister und Kunden beim Verfolgen ihrer Ziele wechselseitig voneinander abhängig sind. Solche Probleme - etwa das Faktum, dass Dienstleistungen immer Dienstleistungsversprechen sind, deren Qualität erst ex post beurteilt werden kann - können allerdings auch innerhalb der Dienstleistungsbeziehung interaktiv bearbeitet werden (Weihrich/Dunkel 2003). Inter-

3 Dabei wird die unterschiedliche Provenienz de beiden Autoren - der Erstautor ist Wirtschaftsingenieur, der Zweitautor Arbeitssoziologe - zwar immer wieder aufscheinen. Beide teilen jedoch ein starkes Interesse an einer erfolgreichen Weiterentwicklung von Dienstleistungswissenschaft und Dienstleistungsforschung und die Überzeugung, dass eine Service Science hierzu gute Chancen bietet.

4 Vgl. z. B. die Beiträge von Stauss (2008); Buhl et al (2008) oder Schneider/Bowen (2010)

$5 \mathrm{Vgl}$. www.3sresearch.de.

6 Bemerkenswert ist, dass dieser Artikel aufgrund der radikal veränderten Sichtweise und der dadurch entstehenden kontroversen Diskussion über fünf Jahre benötigte, um im Journal of Marketing als hochrangiger Fachzeitschrift erscheinen zu können. Der Ansatz von Vargo/Lusch wurde intensiv und kritisch diskutiert - unbestritten ist, dass er wie kaum ein anderer Beitrag die Agenda der jüngeren Diskussionen im Bereich der Service Science bestimmt hat.

7 Vgl. hierzu das Projekt "PiA - Professionalisierung interaktiver Arbeit" (www.interaktive-arbeit.de) im Rahmen des aktuellen BMBF-Förderschwerpunktes „Dienstleistungsqualität durch professionelle Arbeit ${ }^{\prime}$. 
aktive Arbeit steht damit im Kernbereich von Dienstleistungsarbeit (Böhle in diesem Heft; Dunkel/Weihrich 2010; Ernst/ Kopp 2011). Von ihrem Gelingen hängen der Erfolg und die Qualität von Dienstleistungen ab. Dabei betont eine sozialwissenschaftliche Sichtweise, dass die Interessen der beteiligten sozialen Akteure nicht per se in produktiver Weise zusammengehen, sondern dass die Herstellung von Kooperation selbst erst einmal erreicht werden muss.

Mit der Fokussierung auf den Aspekt der gemeinsamen Wertschöpfung schließt die Service Science an traditionelle Betrachtungsweisen in der wissenschaftlichen Beschäftigung mit Dienstleistungen an. Sie erweitert diese Betrachtungsweisen jedoch zum einen um die Vernetzung wirtschaftlicher, technischer und sozialer Aspekte und Gestaltungsoptionen. Zum anderen verzichtet sie auf die bekanntermaßen nicht trennscharfe Abgrenzung zwischen Produkten und Dienstleistungen bzw. sekundärem und tertiärem Sektor. Stattdessen integriert sie die Betrachtung des gesamten Kontinuums von Leistungen von Produkten über hybride Leistungen (Böhmann/Krcmar 2007) bis hin zu Dienstleistungen, indem sie auf die genannten dienstleistungsspezifischen Komponenten abhebt.

\section{Die Konsequenz: von der Dienstleistung zum Dienst- leistungssystem}

Dienstleistungswissenschaft (Service Science) im oben beschriebenen Sinne geht damit über die Dienstleistungsdyade hinaus; sie beschäftigt sich mit der Beschreibung, Erklärung und Gestaltung sozio-technischer Systeme, in denen mehrere Partner durch Leistungsbeziehungen verbunden sind und in gegenseitiger Abstimmung durch den Einsatz der bei allen Partnern vorhandenen Ressourcen gemeinsam Wert für die jeweiligen Partner und/oder das gesamte System schöpfen (Satzger/Ganz et al. 2010). In solchen Service-Systemen (Maglio et al. 2010) sind technologische und soziale Komponenten eng miteinander verwoben:

Service-Systeme als technische Systeme: Offensichtlich steht und fällt die Bedeutung einer Dienstleistungswissenschaft mit den
Möglichkeiten, Interaktion zwischen zwei oder mehreren Partnern herbeizuführen und/oder Leistungen zu individualisieren. Beides wird gerade durch den Einsatz sich rasant innovierender Technologien, insbesondere der Informations- und Kommunikationstechnologie (IKT), befördert - nicht zuletzt durch die Entwicklung des Internets seit den frühen 1990er Jahren: IKT treibt einerseits die wirtschaftliche Bedeutung digital erbringbarer Leistungen und ermöglicht andererseits eine zunehmend (globale) Vernetzung von Partnern. Dadurch stellt sie nicht nur intensivere und zahlreichere Beziehungen zwischen Anbietern und Nachfragern her; sie ändert auch traditionelle Rollenverteilungen von Leistungsgebern und -nehmern. So werden z. B. Kunden vermehrt in den Innovationsprozess einbezogen (Hippel 2007; Möslein/ Kölling 2007; Reichwald/Piller 2006) oder Produzenten- und Kundenrollen sind gar nicht mehr unterscheidbar - wie z. B. im modernen Web-2.0-Ansatz der WikipediaEnzyklopädie. Die Bedeutung der IKT für eine Dienstleistungswissenschaft erklärt auch, warum der Aufschwung der Dienstleistungswissenschaft gerade in den letzten Jahren eingesetzt hat - erst jetzt werden die Potenziale deutlich und greifbar. Und sie erklärt, warum immer wieder Interdisziplinarität gefordert wird: vor allem die Kombination ökonomischer, sozialwissenschaftlicher und technischer Ansätze vermag neue, relevante und anwendungsorientierte Forschungsergebnisse hervorzubringen.

Service-Systeme als soziale Systeme: Service-Systeme lassen sich nicht allein als technische oder ökonomische Systeme begreifen, die über Operations and IT Management u.a.m. zu optimieren wären - Service-Systeme sind immer auch soziale Systeme. Denn in Service-Systemen spielen Menschen - Kunden, Beschäftigte, Führungskräfte - eine konstitutive Rolle. Das Dienstleistungsgeschehen stellt sich als ein komplexer sozialer Prozess dar, der stark durch kulturelle Faktoren geprägt ist (Schneider/Bowen 2010). So haben Kunden nicht nur spezifische Erwartungen an Dienstleistungsunternehmen, sondern tief liegende Bedürfnisse, die auf Sicherheit, Wertschätzung und Gerechtigkeit abzielen und die von Dienstleistungsunternehmen nicht verletzt werden sollten (ebd.). Beschäftigte mit Kundenkontakt wiederum finden sich in der typischen Situation der Grenzstellenarbeit, in der sie
Anforderungen des Unternehmens und Kundenbedürfnisse miteinander vermitteln müssen (vgl. auch Frenkel et al. 1999 oder Holtgrewe/Kerst 2002). Eine Betrachtung von Service-Systemen als soziale Systeme legt des Weiteren nahe, den Kanon empirischer Methoden über quantitative Verfahren auf qualitative Fallstudien zu erweitern. Gummesson (2010) verbindet beispielsweise einen solchen Fallstudienansatz mit der Netzwerktheorie. Dabei besteht der Bezugspunkt für ihn in dem Gegenstand der Dienstleistung, nämlich dem Problem, um dessen Lösung herum sich ein Netzwerk von Akteuren und Prozessen bildet. Im Gegensatz zur, wie er das ironisch nennt, „survey-dominant Logic“ des Mainstreams ließe sich so die Komplexität, die Dienstleistungen innewohne, sehr viel besser erfassen.

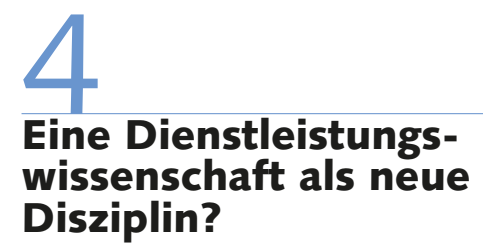

Stauss (2008) hat die Probleme, mit denen die Etablierung einer Service Science konfrontiert ist, systematisch aufbereitet. Dabei begrüßt er zwar den Versuch, Forschung zu Dienstleistungen, wie sie in vielen disziplinären Kontexten geleistet wird, als Dienstleistungsforschung kenntlich zu machen. Die bisherigen Entwicklungen unter der Überschrift Service Science weisen nach seiner Bestandsaufnahme jedoch einige Defizite auf: Zwar hält die Service Science einen interdisziplinären Ansatz für notwendig, bislang dominieren jedoch Ansätze aus dem Dienstleistungsmarketing. Damit geht eine Fokussierung auf ein Erkenntnisinteresse einher, das auf die Lösung von Managementproblemen ausgerichtet ist. Grundlagentheoretische Forschungsinteressen oder Untersuchungen im Sinne von Kunden, Beschäftigten oder Non-Profit-Organisationen fehlen hingegen weitgehend. Stauss konstatiert des Weiteren, dass der Dienstleistungsbegriff möglicherweise zu weit gefasst ist: Was ist der spezifische Gegenstand einer Service Science, wenn im Zuge der Tertiarisierung tendenziell alles zu Dienstleistungen wird? Schließlich sieht er erhebliche Barrieren im akademischen System selbst, die den Aufbau einer neuen Disziplin der Dienstleistungswissenschaft erschweren. 
Damit ist die Frage verbunden, ob die Reise zu einer neuen grundständigen Disziplin gehen soll - oder (zunächst) zu neuen Formen der Zusammenarbeit zwischen Disziplinen sowie zwischen Wissenschaft und Dienstleistungswirtschaft. Nach Meinung der Autoren dieses Artikels wäre bereits viel gewonnen, wenn man dem zuletzt genannten, etwas bescheideneren Ziel näher käme. Hierzu wäre es allerdings notwendig, die multimethodische und interdisziplinäre Ausrichtung der Service Science noch weiter zu entfalten, um die ganzheitliche Analyse und Gestaltung sozio-technischer Service Systeme voranzutreiben. Beispielhaft würde eine Stärkung sozialwissenschaftlicher Perspektiven zusätzliche Kontexte eröffnen und der Service Science zugänglich machen:

- den arbeitsweltlichen Kontext der Arbeitskräfte, die im Rahmen von Dienstleistungssystemen tätig sind;

- den lebensweltlichen Kontext von Kunden, die Dienstleistungen im Rahmen ihrer alltäglichen Lebensführung nutzen und selbst an der Realisierung von Dienstleistungen beteiligt sind;

- den unternehmensübergreifenden Kontext von Kooperationsnetzwerken und Wertschöpfungsketten, der mit vielfältigen Herausforderungen (etwa im Bereich interkultureller Zusammenarbeit in globalisierten Kooperationsbeziehungen) und Potenzialen (wie der Exportfähigkeit von Dienstleistungen) verbunden ist;

- den gesellschaftlichen Kontext, in dessen Rahmen Dienstleistungen bestimmte gesellschaftliche Funktionen erfüllen, in dem es aber auch zu Reibungen, zu Brüchen und Auseinandersetzungen zwischen unterschiedlichen Interessengruppen kommen kann.

Eine Integration sozialwissenschaftlicher Ansätze in den Bereich der Service Science steht bislang noch aus, könnte aber in beide Richtungen positive Wirkungen entfalten. Sie könnte auf der einen Seite der Weiterentwicklung der Service Science dienlich sein, da diese in die Lage versetzt würde, ihre zentrale These der gemeinsamen Wertschöpfung soziologisch zu schärfen. Und sie würde auf der anderen Seite beispielsweise die bislang vorliegenden Ansätze zur interaktiven Arbeit (Weihrich/Dunkel 2003), zur Interaktionsarbeit (Böhle/Glaser 2006) oder zur dialogisch-interaktiven Erwerbsar- beit (Hacker 2009), die noch stark auf das Grundmodell einer dyadischen Dienstleistungsbeziehung zwischen Anbieter und Kunden ausgerichtet sind, für konzeptionelle Erweiterungen wie etwa die Einbettung der Interaktion in komplexere Service-Systeme öffnen.

Eine eigene Dienstleistungswissenschaft wird auf alle Fälle nicht im Elfenbeinturm oder am Reißbrett begründet. Wie bei der Entstehung anderer Disziplinen, wie z. B. der Informatik oder den Arbeitswissenschaften, werden die Anwendungspotenziale den Druck nach Forschungsergebnissen erzeugen. Dann werden auch Konzepte, Methoden und Instrumente entstehen, die über bestehende Disziplinen hinausgehen und eine eigenständige Fachrichtung entstehen lassen. Pragmatisch ist auch weniger die Frage interessant, ob es sich um eine eigene Wissenschaft oder ein Anwendungsfeld handelt, als vielmehr das Ziel, die neue Perspektive wahrzunehmen, sich mit ihr zu beschäftigen und das ihr inhärente Innovationspotenzial zu realisieren. Einige der dann entstehenden Konsequenzen werden im nächsten Abschnitt beleuchtet.

\section{Dienst \\ Dienstleistungswissen- schaft als Nukleus von Innovation}

Offensichtlich bedingen die vorgestellten Konsequenzen der neuen Sichtweise eine Reihe von Veränderungen. Warum sollte man sich diesen unterziehen, wie diese treiben und welche Vorteile entstünden für einzelne Zielgruppen?

\subsection{BILDUNGS- „PFUND“ IN DER INDUSTRIELLEN REVOLUTION 2.0}

In der industriellen Revolution ergaben sich - beginnend im England der zweiten Hälfte des 18. Jahrhunderts - für Individuen, Unternehmen und Volkswirtschaften dramatische soziale und wirtschaftliche Veränderungen. Diese hatten ihren Ursprung zum einen in der zunehmenden Freisetzung von Arbeitskraft durch Bevölkerungswachstum und Landflucht (aufgrund der Auflösung der Allmenderechte), zum anderen darin, dass wissenschaftliche Erkenntnisse, v. a. in den Ingenieurwissenschaften, erzielt, erprobt und umgesetzt werden konnten. Das Wirtschaften mit Produkten war als solches natürlich nicht neu, wurde aber durch die neuen Methoden und Verfahren auf ein gänzlich neues Niveau gehoben. Diejenigen Wettbewerber, die als Erste die wissenschaftlichen Erkenntnisse erlangten und einzusetzen wussten, haben nachhaltig davon profitiert.

Wir dürfen mit einiger Berechtigung davon ausgehen, dass wir erneut an der Schwelle einer solchen "Revolution“" stehen, die auch diesmal ein schon lange existierendes Phänomen - die in Dienstleistungen inhärente gemeinsame Wertschöpfung dramatisch an Bedeutung gewinnen lässt, indem eine wesentliche Technologie, die IKT, entsteht und katalysiert (Satzger 2010). Eingedenk der Veränderungen, die wir durch sie schon in den letzten 20-30 Jahren erfahren haben, dürfte diese „Dienstleistungs-Revolution“ (Karmarkar 2004) weit schneller vonstattengehen als die oben erwähnte Industrielle Revolution. Will man als Volkswirtschaft an ihren Potenzialen teilhaben, empfiehlt sich daher dringend ein schnelles und entschlossenes Vorgehen, um die dafür benötigten Kenntnisse zu erlangen, die eigenen Mitglieder mit diesen auszustatten und den Transfer in die Praxis in der Breite voranzutreiben. Und es empfiehlt sich, von Anfang an die sozialen und gesellschaftlichen Implikationen zu beachten und aktiv zu gestalten, um soziale Probleme, wie sie die Transformationsprozesse der Industriellen Revolution begleiteten, zu vermeiden. Insofern wird es nicht nur darum gehen, ökonomisch erfolgreich zu sein, sondern die Dienstleistungsgesellschaft auch im Sinne von Gerechtigkeit und Nachhaltigkeit zu gestalten.

Die Ausgestaltung einer Dienstleistungswissenschaft wird also kritisch sein: Wir stehen hier erst am Beginn (Spath/ Ganz 2008). Deutschland hat jedoch exzellente Voraussetzungen, in dieser Industriellen Revolution 2.0 voranzugehen: Innovationsorientierung, ausgeprägte Systemkompetenz, Erfahrungen im Technologietransfer, gezielte Forschungsförderung und eine Vorreiterrolle in der Dienstleistungsforschung - z. B. im „Service Engineering" (Luczak et al. 2004; Bullinger/ Scheer 2006) - bieten eine solide Startbasis. Angesichts der schnellen Entwicklung, die hier zu erwarten und global auch schon zu beobachten ist, ist konsequentes und entschlossenes Handeln dennoch ein Muss, wie wir anhand der folgenden Überlegungen beispielhaft zeigen. 
(1) Globalisierung: Die Gewichte der weltweit erschließbaren Märkte werden sich in den nächsten Jahren dramatisch verschieben, ${ }^{8}$ während gleichzeitig die Möglichkeiten zunehmen, über die oben diskutierten Informations- und Kommunikationstechnologien an solchen Märkten teilzuhaben (Satzger 2008). Eine Vernachlässigung „dienstleistungsorientierten“ Exports könnte Deutschland den Anschluss im internationalen Wettbewerb verpassen lassen.

(2) Professionalisierung der Ausbildung: Offensichtlich bedingen Dienstleistungsinnovationen vor allem auch die Zusammenführung technologischer und wirtschaftlicher Kenntnisse sowie sozialer Fähigkeiten. Die Schaffung und Bewerbung entsprechender Ausbildungsprofile, ob in der beruflichen Bildung oder dem Hochschulbereich, erscheinen hier überaus wichtig. Die demografische Entwicklung in Deutschland, der Nachwuchsmangel in MINT-Fächern, ${ }^{9}$ die mangelnden Anreize für Interdisziplinarität in wissenschaftlichen Karrieren und in der Forschungsförderung sowie eine Abschottung der Wissenschaft von der Wirtschaft zulasten eines gesunden Innovationsklimas stellen zentrale Gefahren dar.

(3) Wertschätzung: Weite Bereiche gerade personenbezogener Dienstleistungen leiden unter mangelnder gesellschaftlicher Wertschätzung. Dabei wird etwa der Bereich der Pflegearbeit in Zukunft noch an Bedeutung gewinnen. Wenn es nicht gelingt, gerade mittlere Qualifikationsebenen in der Dienstleistungsarbeit entscheidend aufzuwerten, wird der zum Teil bereits jetzt bestehende Mangel an Fachkräften dramatische Ausmaße annehmen.

\subsection{MASSNAHMEN ZUR FÖRDERUNG}

Was also können Politik, Wirtschaft und Wissenschaft konkret tun, um die Etablierung einer Dienstleistungswissenschaft voranzubringen? Die vorangegangenen Ausführungen haben gezeigt, dass es gute Gründe gibt, sich mit der Implementierung einer Service Science auseinanderzusetzen. Erfahrungen mit der Gründung vergleichsweise junger Wissenschaftsdisziplinen wie der "Life Sciences“ oder der „Umweltwissenschaften“ zeigen jedoch, dass die Implementierung einer neuen Wissenschaft schon aufgrund der Viel- zahl involvierter heterogener Akteure einen langen und schwer steuerbaren Prozess darstellt. Gleichwohl soll an dieser Stelle versucht werden, Maßnahmenpakete zu skizzieren, die einen solchen Implementierungsprozess anregen und unterstützen. Auf Basis des derzeitigen Wissensstandes hat eine Expertengruppe im Auftrag der „Taskforce Dienstleistungen“ drei Gruppen von Handlungsempfehlungen identifiziert, die darauf abzielen, die Implementierung einer Service Science aktiv zu unterstützen (Satzger/Ganz et al. 2010):

(1) Kommunikation der Vision einer Service Science und die Vernetzung von Akteuren: Zunächst gilt es, bei den relevanten Zielgruppen ein hinreichendes Bewusstsein für die Themengebiete und die Notwendigkeit einer Service Science zu schaffen. Hierzu bedarf es der Entwicklung eines gemeinsamen Grundverständnisses dieser Disziplin, einer einheitlichen Sprache und Terminologie sowie deren flächendeckender Verbreitung - z. B. über Positionspapiere, Disziplinen übergreifende Arbeitskreise, Verbände oder Konferenzen. Um den zunehmenden wirtschaftlichen und wissenschaftlichen Anforderungen einer Service Science gerecht zu werden, ist zudem eine interdisziplinäre Ausrichtung der Lehre zwingend erforderlich. Dies kann z.B. durch fakultätsübergreifende Lehrkonzepte oder die Einrichtung spezifischer interdisziplinärer Studiengänge erreicht werden. Ebenso bietet das wachsende Feld der akademischen Weiterbildung, wie z. B. das Angebot von unternehmensspezifischen Lehrgängen, Zertifikatslehrgängen bis hin zu Executive MBA/M.Sc.-Programmen, eine Vielzahl von Möglichkeiten, das Thema Service Science verstärkt auch in die unternehmerische Landschaft zu tragen. Um diese Chance nutzen zu können, sind Wirtschaft und Wissenschaft gefragt, Kooperationen zu etablieren bzw. zu intensivieren - wie sie z. B. am KIT mit Shared Professorships aus der Exzellenzinitiative des BMBF aktiv gefördert werden. Zudem ist die Wirtschaft gefordert, die Schaffung und Kommunikation neuer Berufsbilder voranzutreiben, um Angebot und Nachfrage zusammenzuführen.

(2) Gründung und Förderung entsprechender Programme und Institutionen: Zwar hängt der Erfolg der Bemühungen zur Implementierung einer Service Science primär vom Engagement der Wissenschaft ab. Dennoch können sowohl Wirtschaft als auch Politik förderliche Rahmenbedingungen schaffen. Das größte Potenzial ist hierbei im Angebot entsprechender Förderprogramme zu sehen, sei es im Rahmen der unternehmerischen Auftragsforschung oder durch die Forschungsförderung seitens des BMBF oder der DFG. Es geht aber nicht allein um ein Mehr an finanziellen Mitteln, sondern auch um neue forschungspolitische Akzente. So sollten z. B. vermehrt Bekanntmachungen entwickelt werden, die die Vergabe von Forschungsgeldern von einem interdisziplinären Dienstleistungsforschungsansatz der Antragsteller abhängig machen. Wichtig wäre zudem, eine verstärkt internationale Vernetzung von Förderaktivitäten zu erreichen.

(3) Institutionalisierung der Vision einer Service Science: Das konsequente Weiterdenken der vorangegangenen Empfehlungen führt zu der Frage, ob und wie die Vision einer Service Science sich auch institutionell an der Schnittstelle von Wissenschaft und Wirtschaft verankern lässt. Eine solche Institution würde unterschiedliche Aufgaben und Zielsetzungen verfolgen: Zum einen ermöglichte sie es den unterschiedlichen Wissenschaften und Disziplinen, die sich mit dienstleistungsspezifischen Frage- und Problemstellungen beschäftigen, mit einer Stimme zu sprechen. Zum anderen fungierte sie als Ansprechpartner und beratende Einrichtung für Politik, Wirtschaft und Verbände, förderte die nationale und internationale Sichtbarkeit der deutschen Service Science und diente als Plattform für den Wissenstransfer zwischen Wirtschaft, Politik und Wissenschaft. Ferner sollte die thematische, methodische und institutionelle Entwicklung der Forschung und Lehre einer Service Science zu ihren Aufgaben gehören, ebenso wie die Förderung des wissenschaftlichen Nachwuchses. Ein vom BMBF gefördertes Projekt untersucht derzeit bereits geeignete Institutionalisierungsoptionen.

\footnotetext{
8 Nach einer Schätzung von PricewaterhouseCoopers machen die E7-Staaten ("Emerging 7“ - Brasilien, Russland, Indien, China, Indonesien, Mexiko, Türkei) heute lediglich $20 \%$ der Wirtschaftskraft der G7-Staaten aus, werden aber bis 2050 um bis zu $25 \%$ darüber liegen (Hawksworth 2006).

9 Mathematik, Informatik, Naturwissenschaft und
} Technik. 


\subsection{POTENZIELLE NUTZNIESSER EINER SERVICE SCIENCE}

Die Debatte um den Aufbau der Service Science wurde bislang vor allem im wissenschaftlichen Bereich (und dort vor allem in bestimmten Disziplinen wie dem Dienstleistungsmarketing oder der Wirtschaftsinformatik) und bei großen internationalen Unternehmen (Jetter et al. 2009) geführt. Wir sind jedoch der Meinung, dass die Potenziale einer integrierten Dienstleistungswissenschaft weit über diese Felder und die dort verorteten Akteure hinausreichen.

Dies betrifft zunächst den Bereich der Wissenschaften selbst. Hier gilt es, wie in diesem Beitrag mehrmals angesprochen, die Interdisziplinarität, die im Grundverständnis der Service Science angelegt ist, umfassender als bislang umzusetzen.

Es betrifft die Unternehmen: Sie können - von Anbietern wissensintensiver IT-Dienstleistungen bis hin zu Anbietern personenbezogener Dienstleistungen von systematischen Erkenntnissen einer Dienstleistungswissenschaft profitieren und diese für das eigene Handeln nutzen. Hier ist etwa an ein systemisches Denken gedacht, das technologische und soziale Aspekte im Zusammenhang begreift, ferner an die Weiterentwicklung von Dienstleistungsangeboten gemäß des Value-in-Use-
Gedankens oder die innovative Gestaltung kooperativer Wertschöpfungsprozesse, die Kunden und Beschäftigte gleichermaßen berücksichtigen.

Nicht zuletzt birgt die Service Science erhebliche Potenziale für die Dienstleistungspolitik und die Ausgestaltung der Dienstleistungsgesellschaft der Zukunft:

- Sie kann über die Analyse von Machtverhältnissen in Dienstleistungsbeziehungen und das Aufdecken spezifischer Belastungen, die mit kooperativen Wertschöpfungsprozessen zusammenhängen, den Veränderungsbedarf soziotechnischer Dienstleistungssysteme benennen.

- Sie kann über die Durchführung entsprechender Forschungsprojekte zur Bearbeitung gesellschaftlicher Probleme (wie etwa des demografischen Wandels) beitragen.

- Sie kann, gerade aufgrund ihrer bereits beeindruckenden internationalen Bedeutung dazu beitragen, der traditionell starken deutschen Industrieforschung eine stärker werdende Dienstleistungsforschung an die Seite zu stellen.

- Sie kann den Rahmen für Bemühungen bilden, eine allgemeine Dienstleistungstheorie zu entwickeln und auf diese Weise helfen, politische Herausforderungen über unterschiedliche Dienstleistungsbranchen hinweg zu benennen.
Die Service Science wird solche Wirkungen aber nur dann erreichen können, wenn sie sich selbst weiter entwickelt. Hierfür sind die bereits beteiligten und die potenziell zu beteiligenden Wissenschaften verantwortlich. Sie sind dies aber nicht alleine. So ist eine Gewerkschaft wie die Vereinte Dienstleistungsgewerkschaft (ver. di), die für Dienstleistungspolitik in einer Vielzahl von Branchen zuständig ist, geradezu prädestiniert dafür, sich nicht nur als Konsument wissenschaftlicher Fortschritte, sondern auch als Akteur an der Debatte zu beteiligen. Ein wichtiger Beitrag könnte aus der Sicht der Autoren darin bestehen, die Service Science dazu anzuhalten, wissenschaftlichen Kriterien (etwa in Bezug auf eine hoch entwickelte Dienstleistungstheorie) und unternehmensbezogenen Kriterien (etwa der Effizienzsteigerung oder der erfolgreichen Platzierung neuer Dienstleistungen auf den Märkten) Kriterien guter Arbeit und nachhaltiger Ressourcenverwendung an die Seite zu stellen. Daraus wiederum lieBen sich, systematischer als dies bislang zu leisten war, Folgerungen für Arbeitsgestaltung und Qualifizierung ziehen, die $\mathrm{zu}$ einer gelungenen Kombination von Technisierung und Humanisierung von Dienstleistungen beitragen.

\section{LITERATUR}

Böhle, F./Glaser, J. (2006): Arbeit in der Interaktion - Interaktion als Arbeit: Arbeitsorganisation und Interaktionsarbeit in der Dienstleistung, Wiesbaden

Böhmann, T./Krcmar, H. (2007): Hybride Produkte: Merkmale und Herausforderungen, in: Bruhn, M./Stauss, B. (Hrsg.): Wertschöpfungsprozesse bei Dienstleistungen, Wiesbaden, S. 239-256

Buhl, H. U./Heinrich, B./Henneberger, M./Krammer, A. (2008): Service Science, in: Wirtschaftsinformatik 50 (1), S. 60-65

Bullinger, H./Scheer, A. (2006): Service Engineering - Entwicklung und Gestaltung innovativer Dienstleistungen, Berlin

Dunkel, W./Bienzeisler, B. (2011): „3sR - Social Science Service Research“ - der Beitrag der sozialwissenschaftlichen Dienstleistungsforschung zu einer aktuellen Debatte, in: Gatermann, I./Fleck, M. (Hrsg.):
Mit Dienstleistungen die Zukunft gestalten, Beiträge der 8. Dienstleistungstagung des BMBF, Frankfurt a. M., S. 353-358

Dunkel, W./Weihrich, M. (2010): Arbeit als Interaktion, in: Böhle, F./ Voß, G./Wachtler, G. (Hrsg.): Handbuch Arbeitssoziologie, Wiesbaden, S. 177-200

Engelhardt, W. H./Kleinaltenkamp, M./Reckenfelderbäumer, M. (1993): Leistungsbündel als Absatzobjekte - Ein Ansatz zur Überwindung der Dichotomie von Sach- und Dienstleistungsobjekten, in: Zeitschrift für betriebswirtschaftliche Forschung 45 (5), S. 395-426 Ernst, G./Kopp, I. (2011): Interaktionsarbeit als zentrales Element der Dienstleistungsinnovation, in: Schröder, L./Urban, H.-J. (Hrsg.): Gute Arbeit. Folgen der Krise, Arbeitsintensivierung, Restrukturierung, Frankfurt a. M., S. $261-273$ 
Frenkel, S. J./Korczynski, M./Shire, K. A./Tam, M. (1999): On the front line: Work organization in the service economy, Ithaca/New York Gummesson, E. (2010): The Future of Service Is Long Overdue, in: Maglio, P./Kieliszewski, C. A./Spohrer, J. C. (Hrsg.): Handbook of Service Science, New York, S. 625-642

Hacker, W. (2009): Arbeitsgegenstand Mensch: Psychologie dialogischinteraktiver Erwerbsarbeit, Lengerich

Hawksworth, J. (2006): The World in 2050 - How big will the major emerging market economies get and how can the OECD compete?, http://www.pwc.com/extweb/pwcpublications.nsf/docid/56DD37D0C 399661D852571410060FF8B/\$file/world2050emergingeconomies.pdf (letzter Zugriff: 7.8.2011)

Hippel, E. von (2007): Horizontal innovation networks - by and for users, in: Industrial and corporate change 16 (2), S. 293-315

Holtgrewe, U./Kerst, C. (2002): Zwischen Kundenorientierung und organisatorischer Effizienz - Callcenter als Grenzstellen, in: Soziale Welt 53 (2), S. 141-160

Institute for Manufacturing, University of Cambridge/International Business Machines Corporation (IfM/IBM) (Hrsg.) (2008): Succeeding through Service Innovation: A service perspective for education, research, business and government, Cambridge

Jacobsen, H. (2010): Strukturwandel der Arbeit im Tertiarisierungsprozess, in: Böhle, F./Voß, G./Wachtler, G. (Hrsg.): Handbuch Arbeitssoziologie, Wiesbaden, S. 203-228

Jetter, M./Satzger, G./Neus, A. (2009): Technologische Innovation und die Auswirkung auf Geschäftsmodell, Organisation und Unternehmenskultur - Die Transformation der IBM zum global integrierten, dienstleistungsorientierten Unternehmen, in: Wirtschaftsinformatik 51 (1), S. 43-52

Karmarkar, U. (2004): Will You Survive the Services Revolution?, in: Harvard Business Review 82 (6), S. 100-108

Kleinaltenkamp, M. (2001): Begriffsabgrenzungen und Erscheinungsformen von Dienstleistungen, in: Bruhn, M./Meffert, H. (Hrsg.): Handbuch Dienstleistungsmanagement. Von der strategischen Konzeption zur praktischen Umsetzung, Wiesbaden, S. 31-52

Korczynski, M./Macdonald, C. L. (Hrsg.) (2009): Service Work. Critical Perspectives, New York

Luczak, H./Reichwald, R./Spath, D. (2004): Service Engineering in Wissenschaft und Praxis: Die ganzheitliche Entwicklung von Dienstleistungen, Wiesbaden

MacLean, A. M. (1899): Two weeks in department stores, in: American Journal of Sociology 4 (6), S. 721-741

Maglio, P. P. (2006): Service systems, service scientists, SSME, and innovation, in: Communications of the ACM 49 (7), S. 81-85

Maglio, P. P./Kieliszewski, C. A./Spohrer, J. C. (Hrsg.) (2010): Handbook of Service Science, New York

Möslein, K. M./Kölling, M. (2007): Interaktive hybride Wertschöpfung als Innovationsstrategie, in: Streich, D./Wahl, D. (Hrsg.): Innovationsfähigkeit in einer modernen Arbeitswelt - Beiträge der Tagung des BMBF, Frankfurt a. M., S. 195-202
Papazoglou, M. P./Georgakopoulos, D. (2003): Service-Oriented Computing, in: Communications of the ACM 46 (10), S. 25-28 Reichwald, R./Piller, F. (2006): Interaktive Wertschöpfung: Open Innovation, Individualisierung und neue Formen der Arbeitsteilung, Wiesbaden

Rust, R. T./Miu, C. (2006): What academic research tells us about service, in: Communications of the ACM 49 (7), S. 49-54

Satzger, G. (2008): Dienstleistungswissenschaft - Anforderungen der Praxis an Dienstleistungsforschung und -lehre, in: Gaterman, I./Fleck, M. (Hrsg.): Technologie und Dienstleistung - Beiträge der 7. Dienstleistungstagung des BMBF, Frankfurt a. M., S. 187-195

Satzger, G. (2010): Service Science - der Schlüssel zur Wettbewerbsfähigkeit in der "Industriellen Revolution 2.0"?, in: Gatermann, I./Fleck, M. (Hrsg.): Mit Dienstleistungen die Zukunft gestalten - Beiträge der 8. Dienstleistungstagung des BMBF, Frankfurt a. M., S. 349-352

Satzger, G./Ganz, W. et al. (2010): Auf dem Weg zu einer Service Science - Perspektiven, Forschungsthemen und Handlungsempfehlungen aus der Sicht einer interdisziplinären Arbeitsgruppe. Bericht der Arbeitsgruppe „Evaluation Service Science" der Taskforce Dienstleistungen Stuttgart, http://www.ksri.kit.edu/Upload/ Publications/92d75a33-9e07-473b-9091-7398a0557f0b.pdf (letzter Zugriff: 7.8.2011)

Schneider, B./Bowen, D. E. (2010): Winning the Service Game: Revisiting the Rules by Which People Co-Create Value, in: Maglio, P. P./ Kieliszewski, C. A./Spohrer, J. C. (Hrsg.): Handbook of Service Science, New York, S. 31-59

Spath, D./Ganz, W. (Hrsg.) (2008): The Future of Services - Trends and Perspectives, München

Spohrer, J./Maglio, P./Bailey, J./Gruhl, D. (2007): Steps Toward a Science of Service Systems, in: IEEE Computer 40 (1), S. 70-77

Stauss, B. (2008): International Service Research - Status Quo, Developments, and Consequences for the Emerging Services Science, in: Stauss, B./Engelmann, K./Kremer, A./Luhn, A. (Hrsg.): Services Science - Fundamentals, Challenges and Future Developments, Berlin, S. $57-70$

Stauss, B./Engelmann, K./Kremer, A./Luhn, A. (Hrsg.) (2008): Services Science - Fundamentals, Challenges and Future Developments, Berlin Teboul, J. (2006): Service is front Stage - Positioning services for value advantage, Basingstoke/New York

Vargo, S. L./Lusch, R. F. (2004): Evolving to a new dominant logic for marketing, in: Journal of Marketing 68 (1), S. 1-17

Vargo, S. L./Lusch, R. F. (2008): From goods to service(s): Divergences and convergences of logics, in: Industrial Marketing Management 37

(3), S. 254-259

Voswinkel, S. (2005): Welche Kundenorientierung? Anerkennung in der Dienstleistungsarbeit, Berlin

Weihrich, M./Dunkel, W. (2003): Abstimmungsprobleme in Dienstleistungsbeziehungen - Ein handlungstheoretischer Zugang, in: Kölner Zeitschrift für Soziologie und Sozialpsychologie 55 (4), S. $758-781$ 